\title{
P04-55 LB. Anti-V3 monoclonal antibodies display broad neutralizing activities against multiple HIV-I subtypes S Zolla-Pazner ${ }^{4}$, T Wrin ${ }^{1}$, MS Seaman ${ }^{2}, \mathrm{X} \mathrm{Yu}^{3}$, B Wood ${ }^{3}$, S Self ${ }^{3}$ and CE Hioe*4
}

Address: ${ }^{1}$ Monogram Biosciences Inc., South San Francisco, USA, ${ }^{2}$ Beth Israel Deaconess Medical Center, Boston, USA, ${ }^{3}$ SCHARP, Seattle, USA and ${ }^{4}$ New York University School of Medicine, New York, USA

* Corresponding author

from AIDS Vaccine 2009

Paris, France. 19-22 October 2009

Published: 22 October 2009

Retrovirology 2009, 6(Suppl 3):P395 doi:10.1 186/1742-4690-6-S3-P395

This abstract is available from: http://www.retrovirology.com/content/6/S3/P395

(C) 2009 Zolla-Pazner et al; licensee BioMed Central Ltd.

\section{Background}

The V3 loop of gp120 was identified as the "principle neutralizing domain" of HIV-1, but because of its high sequence variability, V3 has been considered an inappropriate target for vaccines. However, V3 is a part of the Env that interacts with the chemokine receptors and therefore, despite its sequence variation, must retain conserved structural features. Nevertheless, the ability of anti-V3 antibodies to neutralize diverse HIV-1 isolates from different subtypes has remained in question.

\section{Methods}

HIV neutralization experiments were conducted in two independent labs to test seven anti-V3 monoclonal antibodies (mAbs) against a total of 98 pseudoviruses (psVs) expressing Env of HIV-1 subtypes A, B, C, D, and AG from acute and chronic patients. MAbs against parvovirus or anthrax antigens were used as negative controls. Statistically significant neutralization was determined based on evaluation of the area under the titration curve (AUC).

\section{Results}

Statistically significant neutralization $(\mathrm{p}<0.001)$ was detected with each anti-V3 mAb against $26-35 \%$ of psVs but not with the irrelevant mAb controls. For many $\mathrm{mAb} /$ $\mathrm{psV}$ combinations, 50\% neutralization was not attained at the highest $\mathrm{mAb}$ concentration tested $(50 \mu \mathrm{g} / \mathrm{ml})$, although dose-response relationships were demonstrated by the neutralization curves, and the AUC values were sig- nificantly above background. All seven mAbs showed statistically significant neutralizing activities against psVs carrying Envs of all subtypes tested. As many as 63\% and $45 \%$ of psVs with subtypes B and C Envs had significant neutralization activity, respectively, by at least one anti-V3 $\mathrm{mAb}$, although among psVs with Env of subtypes A and D, only one or two in each subtype (10-20\%) were sensitive to the anti-V3 mAbs. Overall, 52 (53\%) of $98 \mathrm{psVs}$ showed significant neutralization activity against one or more anti-V3 mAbs.

\section{Conclusion}

While the neutralization potency of individual anti-V3 mAbs is generally low, anti-V3 mAbs display both intraand inter-subtype neutralizing activities. 